BONN-TH-2007-05

\title{
Focus Point SUSY at the LHC Revisited
}

\author{
Siba Prasad Das ${ }^{1}$ \\ Physikalisches Institut der Universität Bonn, Nußallee 12, D-53115 Bonn, Germany \\ Amitava Datta ${ }^{2}$ \\ Department of Physics, Jadavpur University, Kolkata 700 032, India \\ Monoranjan Guchait ${ }^{3}$ \\ Tata Institute of Fundamental Research, Homi Bhaba Road, Mumbai 400 005, India \\ Manas Maity ${ }^{4}$ and Siddhartha Mukherjee ${ }^{5}$ \\ Department of Physics, Visva-Bharati, Santiniketan 731 325, India
}

\begin{abstract}
The estimation of the backgrounds for gluino signals in focus point supersymmetry is extended by including the backgrounds from the production of four third generation quarks in the analysis. We find that these backgrounds are negligible if one uses the strong selection criteria proposed in the literature (including this analysis) for heavy gluino searches. Softer selection criteria often recommended for lighter gluino searches yield backgrounds which are small but numerically significant. We have also repeated the more conventional background calculations and compared our results with the other groups. We find that the size of the total residual background estimated by different groups using different event generators and hard kinematical cuts agree approximately. In view of the theoretical uncertainties in the leading order signal and background cross sections mainly due to the choice of the QCD scale, the gluino mass reach at the LHC cannot be pinpointed. However, requiring a signal with $\geq 3$ tagged b-jets ( instead of the standard choice of $\geq 2$ ) it is shown that gluino masses close to $2 \mathrm{TeV}$ can be probed at the LHC for a range of reasonable choices of the QCD scale for an integrated luminosity of $300 \mathrm{fb}^{-1}$.
\end{abstract}

PACS no: 11.30.Pb, 12.60.Jv, 14.80.Ly, 95.35.+d

\footnotetext{
${ }^{1}$ spdas@th.physik.uni-bonn.de

2adatta@juphys.ernet.in

${ }^{3}$ guchait@tifr.res.in

${ }^{4}$ manas@vbphysics.net.in

${ }^{5}$ iam_siddhartha_mukherjee@yahoo.co.in
} 


\section{Introduction}

Supersymmetry (SUSY) is one of the most elegant extensions of the Standard Model (SM) which naturally stabilizes the Higgs boson mass even in the presence of a very energy scale like the grand unification scale $\left(\mathrm{M}_{\mathrm{G}}\right)[1]$. This occurs due to the cancellation of the quadratic divergences among loop diagrams involving SM particles and their superpartners (sparticles). This cancellation, however, looses much of its attractive naturalness and smacks of fine tuning if the sparticles are much heavier than the corresponding particles. Thus SUSY models with sparticle masses much larger than $1 \mathrm{TeV}$ are usually disfavored.

On the other hand it is well known that if the scalar superpartners of the fermions belonging to the first two generations in the SM are very heavy with masses in the multi$\mathrm{TeV}$ region then several problems can be avoided without further unnatural adjustment of the parameters. Firstly in the most general minimal supersymmetric extension of the Standard Model (MSSM) there are unacceptably large loop induced flavor changing neutral currents unless the masses of the first two generations of squark are assumed to be degenerate to a very good approximation without a symmetry underlying this degeneracy. This SUSY flavor problem becomes less severe if the above loops are suppressed by the masses of the heavy scalars. Secondly SUSY models with complex soft breaking parameters have potentially dangerous large loop induced CP violating contributions to the electric dipole moments of various particles in general. This SUSY CP problem is also tamed if the scalars are very heavy. Therefore models with very heavy scalars without sacrificing the naturalness criterion are indeed welcome.

All the above attractive features are realized in the focus point (FP)/ hyperbolic branch region [2, 3] of the minimal supergravity(mSUGRA) model[4]. The SUSY flavor and CP problems become less severe [5]. The magnitude of $\mu$, the higgsino mass parameter, which is fixed by the radiative electroweak symmetry breaking ( REWSB ) condition, also turns out to be $\sim m_{Z}$ as required by naturalness $[2,6]$.

The low value of $\mu$ thus obtained has another important implication. The lightest supersymmetric particle (LSP) which is the lightest neutralino $\left(\widetilde{\chi}_{1}^{0}\right)$ over most of the parameter space, is usually a mixture of the electroweak gauginos and Higgsinos. However, for small $\mu$ the higgsino component in $\widetilde{\chi}_{1}^{0}$ dominates. In this case $\widetilde{\chi}_{1}^{0}$ very efficiently annihilates into gauge boson pairs leaving open the attractive possibility that LSP could be a viable dark matter candidate $[7,8,9,10]$. In fact it is now widely acknowledged that the focus point region of the mSUGRA model leads to a dark matter density compatible with the latest WMAP data [11]. Thanks to the above interesting features the collider signatures of focus point supersymmetry have attracted wide attention $[12,13,14,15,16$, $17,18,19]$.

Although the scalar quarks are rather heavy in this model, naturalness arguments imply that the gluinos must be necessarily light [2]. Thus the gluinos are the lightest strongly interacting sparticles within the striking range of the LHC. Inspite of the fact that the squarks belonging to the first two generations are indeed very heavy, the lighter 
squark mass eigenstates belonging to the third generation, though significantly heavier than the gluino, may be relatively light at the weak scale $M_{W}$. This is partly due to the renormalization group ( $\mathrm{RG}$ ) evolution from $M_{G}$ to $M_{W}$ driven by large Yukawa couplings and partly due to mixing effects in the mass matrices ( see, e.g., [12] Eqns.3 - 11). As a result the gluino preferentially decays into third generation quarks via three body modes mediated by the relatively light squarks. Therefore dominant decay modes of the gluino are

$$
\widetilde{\mathrm{g}} \rightarrow \mathrm{f}_{1} \overline{\mathrm{f}_{1}}+\mathrm{X}, \quad \widetilde{\mathrm{g}} \rightarrow \overline{\mathrm{t}} \mathrm{b}(\mathrm{t} \overline{\mathrm{b}})+\mathrm{Y}
$$

where $\mathrm{f}_{1}=\mathrm{t}$ or $\mathrm{b}, \mathrm{X}=\tilde{\chi}_{\mathrm{i}}^{0}$ (the ith neutralino), $\mathrm{i}=1-4$ and $\mathrm{Y}=\tilde{\chi}_{\mathrm{j}}^{ \pm}$(the jth chargino), $\mathrm{j}=1,2$. Hence the signals of gluino decay at the LHC will be rich in b-jets [12]. In addition one or more reconstructed $\mathrm{W}$ bosons in the final state may also help SUSY search via this channel.

The size of the signal involving involving $\geq 3$ tagged b-jets (introduced mainly to control the QCD background) and various number of leptons was estimated by a parton

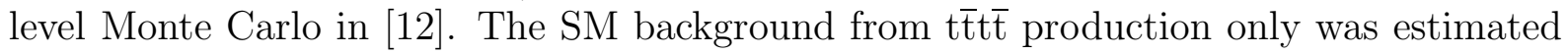
to be small after imposing a strong missing transverse energy $\mathrm{E}_{\mathrm{T}}$ cut.

Subsequently the LHC signal of focus point supersymmetry have been studied by several groups using simulations beyond the naive parton level [13] - [19]. Some of these works required $\geq 2$ tagged b-jets in the final state for efficient background rejection. These works will be briefly reviewed in a later section. However, none of these works considered the background from $f_{1} \bar{f}_{1} f_{2} \bar{f}_{2}$ production, where $f_{i}=\mathrm{t}$ or b. Apriorily, however, some of these backgrounds with production cross sections larger than the gluino pair production cross section( see section 2) by several orders of magnitude seem to be potentially dangerous. Moreover, these backgrounds are rich in b-jets. Hence b-tagging which effectively suppresses light flavor QCD events may not be very effective.

The purpose of this paper is to study the above backgrounds systematically (see section 2) along with a thorough study of the signal and other backgrounds. We have calculated the new background cross sections using CalcHEP[20] v2.4.5, ALPGEN [21] v2.1.1 and MadGraph [22] v4.1. The results agree fairly well. The parton level events are then interfaced with Pythia [23] v6.4.8 for implementing hadronization, fragmentation and a toy detector simulation and analyzed with suitable selection criteria. We shall also study the response of these backgrounds to the cuts used by other groups $[18,19]$. We next study the dominant QCD and tE backgrounds as well as the signal using our selection criteria and compare and contrast the results with other published works ( see section 2 ). In order to facilitate the comparison we required like other groups $\geq 2$ b-jet tags. Finally the consequences of selecting a signal with a richer b-jet content as suggested in [12] are examined. Our conclusions and future outlooks will be summarized in section 3 . 


\section{Signals of Focus Point SUSY at LHC and the Back- grounds}

We begin by briefly reviewing some of the earlier works. Baer et al.[13] scanned the focus point region of the mSUGRA parameter space consistent with the WMAP data. The LHC reach in channels with m-jets $+n$-leptons $+\mathrm{E}_{\mathrm{T}}$ was then examined. It was concluded that the part of the focus point region corresponding to gluino masses $<1.8 \mathrm{TeV}$ is within the reach of LHC. The suppression of the SM background due to light flavor QCD, t $\bar{t}$ etc was considered in estimating the mass reach. However, one of the key features of the signal in the focus point region, namely the large number of b-jets in the final state was not utilized in improving the signal to background ratio.

Subsequently Mercadante et al.[15] estimated that requiring final states with tagged b-jets as suggested in [12] and by adjusting the kinematical cuts the gluino mass reach can be improved by about $20 \%$. However, the potential backgrounds from four heavy flavor production mentioned in the introduction were not included in either of the above analyses. The viability of SUSY signals in the regions of the parameter space consistent with dark matter data was also studied by the CMS collaboration [16]. This analysis included only a part of the $\mathrm{FP}$ region $\left(m_{0}<2 \mathrm{TeV}\right)$ and the above potential backgrounds were neglected.

A few points (FP1 - FP5) in the FP region allowed by the WMAP data from [17] are shown in Table 1. All masses and mass parameters in this paper are in $\mathrm{GeV}$ unless otherwise stated. The other mSUGRA parameters are $A_{0}=0, \tan \beta=30$ and $\operatorname{sign}(\mu)>0$. For each point the gluino mass and the lowest order gluino pair production cross section (in $\mathrm{fb}$ ) for two choices of the QCD scale (see the last two columns in Table 1) are also shown. The first choice yielding smaller cross sections will be subsequently referred to as the conservative choice. The second choice, motivated by the fact that at this scale the leading and next to leading order production cross sections almost agree [24], is the optimistic choice. It is to be noted that we have considered gluino masses near the LHC search limit only. The gluino pair production cross section in the leading order is computed by CalcHEP. The expected sharp fall of the cross section for gluino masses near the kinematic limit is quite apparent.

From the dominant gluino decay modes discussed in the introduction it is clear that a typical final state will consist of four third generation quarks and their decay products. Additional quarks, leptons and missing energy would come from the decays of heavier charginos and neutralinos also produced in gluino decays. Thus the multiplicity of jets and hard isolated leptons in the signal would be large. Keeping this in mind the criteria listed below for selecting ( rejecting) the signal ( the background) events have been formulated. It should be noted that instead of separately requiring large number of jets and hard isolated leptons ( henceforth jets and isolated leptons will be collectively called objects ) we require the total number objects in the final state to exceed nine.

$$
\text { - } \mathrm{N}_{\text {jet }} \geq 6 \text { and } \mathrm{N}_{\text {obj }} \geq 9 \text {. }
$$


- $\mathrm{N}_{\mathrm{btag}} \geq 2$ and one of the tagged jets with $\mathrm{E}_{\mathrm{T}} \geq 300 \mathrm{GeV}$.

- $\mathrm{E}_{\mathrm{T}} \geq 300 \mathrm{GeV}$ where $\mathrm{E}_{\mathrm{T}}$ has been computed using all visible objects in an event.

- $\mathrm{M}_{\mathrm{eff}}=\mathrm{E}_{\mathrm{T}}+\sum_{\mathrm{obj}} \mathrm{E}_{\mathrm{T}} \geq 2000 \mathrm{GeV}$.

It may be noted that at this stage the constraint on $N_{b t a g}$ is similar to the one employed by other analyses. We shall show below that requiring more tagged b-jets in the final state as suggested in [12] improves the signal vis a vis the background.

We begin the analysis by estimating the backgrounds from production of four heavy flavors in different combinations. This will be followed by the simulation of more conventional backgrounds and the signal also studied by other groups. The production cross sections computed by CalcHEP, ALPGEN and MadGraph are presented in Table 2. We have set the following input values at the weak scale $\left(\mathrm{M}_{\text {weak }}\right): \alpha_{\mathrm{em}}=\frac{1}{127.934}, \alpha_{\mathrm{s}}=0.1172$, $\mathrm{m}_{\mathrm{t}}($ pole $)=175.0[25], \mathrm{m}_{\mathrm{b}}\left(\mathrm{m}_{\mathrm{b}}\right)=4.25 \mathrm{GeV}$.

In our calculation we use the CTEQ5L [27] parton distribution functions (PDF). The appropriate loop corrected values of strong coupling constant at the renormalization scale is calculated on the fly during the convolution with the PDF. To avoid the generation of unwanted events the following nominal cuts are applied only on the bottom quarks: $\mathrm{p}_{\mathrm{T}}^{\mathrm{b}}>10 \mathrm{GeV}, \eta(\mathrm{b})<5.0$ and $\Delta \mathrm{R}(\mathrm{b}, \mathrm{b})>0.3$ where $\Delta \mathrm{R}=\sqrt{\Delta \eta^{2}+\Delta \phi^{2}}$.

\begin{tabular}{llll}
\hline \hline & & \multicolumn{2}{c}{ QCD scale } \\
\cline { 3 - 4 } FP point $\left(\mathrm{m}_{0}, \mathrm{~m}_{1 / 2}\right)$ & $\mathrm{m}_{\tilde{\mathrm{g}}}$ & $\sqrt{\hat{\mathrm{s}}}$ & $\mathrm{m}_{\tilde{\mathrm{g}}} / 2$ \\
& & & \\
\hline FP1 $(3700.0,700.0)$ & 1751.3 & 1.18 & 2.37 \\
FP2 $(3975.0,730.0)$ & 1824.7 & 0.81 & 1.63 \\
FP3 ( 3975.0, 790.0 $)$ & 1950.0 & 0.39 & 0.80 \\
FP4 $(4130.0,833.0)$ & 2047.4 & 0.24 & 0.48 \\
FP5 $(4235.0,866.0)$ & 2119.8 & 0.16 & 0.33 \\
& & & \\
\hline \hline
\end{tabular}

Table 1: Cross-sections (in fb) for $\mathrm{pp} \rightarrow \widetilde{\mathrm{g}} \widetilde{\mathrm{g}}$ at LHC using CalcHEP v2.4.5 for different FP scenarios and two choices of the QCD scale (see the last two columns). The masses and mass parameters are in $\mathrm{GeV}$.

In Table 2 we demonstrate the variation of the cross sections with the QCD scale. There is reasonable agreement between different generators at the same scale. As expected there is significant scale dependence in these leading order cross sections. However, in spite of this uncertainty some of the background processes have cross sections several orders of magnitude larger than that of the signal, which is typically a few fb for $\mathrm{m}_{\widetilde{\mathrm{g}}}$ near the LHC search limit (see Table 1). Thus it is worthwhile to see the response of these 


\begin{tabular}{|c|c|c|c|c|}
\hline Process & Generator & $0.5 \sqrt{\hat{\mathrm{S}}}$ & $\sqrt{\hat{\mathrm{S}}}$ & $2.0 \sqrt{\hat{\mathrm{S}}}$ \\
\hline \multirow[t]{3}{*}{$\mathrm{t} \overline{\mathrm{t} t \overline{\mathrm{t}}}$} & MadGraph & 4.38 & 2.82 & 1.93 \\
\hline & ALPGEN & 4.42 & 2.90 & 1.96 \\
\hline & CalcHEP & - & 2.89 & - \\
\hline \multirow[t]{3}{*}{$\mathrm{t} \overline{\mathrm{tb}} \overline{\mathrm{b}}$} & MadGraph & $2.62 \times 10^{3}$ & $1.76 \times 10^{3}$ & $1.14 \times 10^{3}$ \\
\hline & ALPGEN & $2.86 \times 10^{3}$ & $1.89 \times 10^{3}$ & $1.29 \times 10^{3}$ \\
\hline & CalcHEP & - & $2.27 \times 10^{3}$ & - \\
\hline \multirow[t]{3}{*}{$\mathrm{b} \overline{\mathrm{b}} \mathrm{b} \overline{\mathrm{b}}$} & MadGraph & $1.20 \times 10^{7}$ & $7.61 \times 10^{6}$ & $5.62 \times 10^{6}$ \\
\hline & ALPGEN & $1.22 \times 10^{7}$ & $8.04 \times 10^{6}$ & $5.52 \times 10^{6}$ \\
\hline & CalcHEP & - & $1.15 \times 10^{7}$ & - \\
\hline
\end{tabular}

Table 2: Production cross-section (fb) for different final states involving four third generation quarks obtained using different generators. Variation of the cross sections with QCD scale is shown in the last three columns.

backgrounds to our choice of the kinematical cuts listed below as well as to the cuts used by similar analyses $[18,19]$. We shall subsequently show that our cuts also lead to a healthy signal size and control other conventional backgrounds quite effectively.

We next simulate the backgrounds listed in Table 2. The parton level unweighted events from ALPGEN has been interfaced with Pythia. As noted earlier our final conclusion regarding the importance of these backgrounds remain unchanged inspite of the sizable uncertainty due to the choice of scale. In order to avoid generation of events unlikely to pass the cuts we have imposed the following conditions on the parton level sub-processes. It should be noted that these conditions are somewhat stronger than those used in Table 2 .

- $t \bar{t} t \bar{t}$ events have been generated with no cut on $t$.

- $t \bar{t} b \bar{b}$ events have been generated with no cut on $\mathrm{t}, \hat{\mathrm{P}_{\mathrm{T}}^{\mathrm{b}}}>20 \mathrm{GeV}$ and $\left|\eta_{\mathrm{b}}\right| \leq 4.5$.

- $\mathrm{b} \overline{\mathrm{b}} \mathrm{b} \overline{\mathrm{b}}$ events have been generated with $\hat{\mathrm{P}_{\mathrm{T}}^{\mathrm{b}}}>50 \mathrm{GeV}$ and $\left|\eta_{\mathrm{b}}\right| \leq 4.5$.

We have used the toy calorimeter simulation (PYCELL) provided in Pythia with the following criteria:

- The calorimeter coverage is $|\eta|<4.5$. The segmentation is given by $\Delta \eta \times \Delta \phi=$ $0.09 \times 0.09$ which resembles a generic LHC detector. 
- A cone algorithm with $\Delta \mathrm{R}=\sqrt{\Delta \eta^{2}+\Delta \phi^{2}}=0.5$ has been used for jet finding.

- $\mathrm{E}_{\mathrm{T}, \min }^{\mathrm{jet}}=30 \mathrm{GeV}$ and jets are ordered in $E_{T}$.

- Leptons $(\ell=\mathrm{e}, \mu)$ are selected with $\mathrm{E}_{\mathrm{T}} \geq 20 \mathrm{GeV}$ and $|\eta| \leq 2.5$.

- No jet should match with a hard lepton in the event.

- A jet with $|\eta| \leq 2.5$ matching with a B-hadron of significant decay length has been marked tagged.

We have implemented jet and lepton $(\ell=\mathrm{e}$ or $\mu$ ) isolation using the following criteria: if there is a jet within $\Delta \mathrm{R}=0.5$ and $\mathrm{E}_{\mathrm{T}}^{\text {jet }} / \mathrm{E}_{\mathrm{T}}^{\ell} \leq 1.2$, the jet is removed from the list of jets, else the lepton is removed from the list of leptons. Single b-jet tagging efficiency in tét has been tuned to $\epsilon_{\mathrm{b}} \simeq 0.5$.
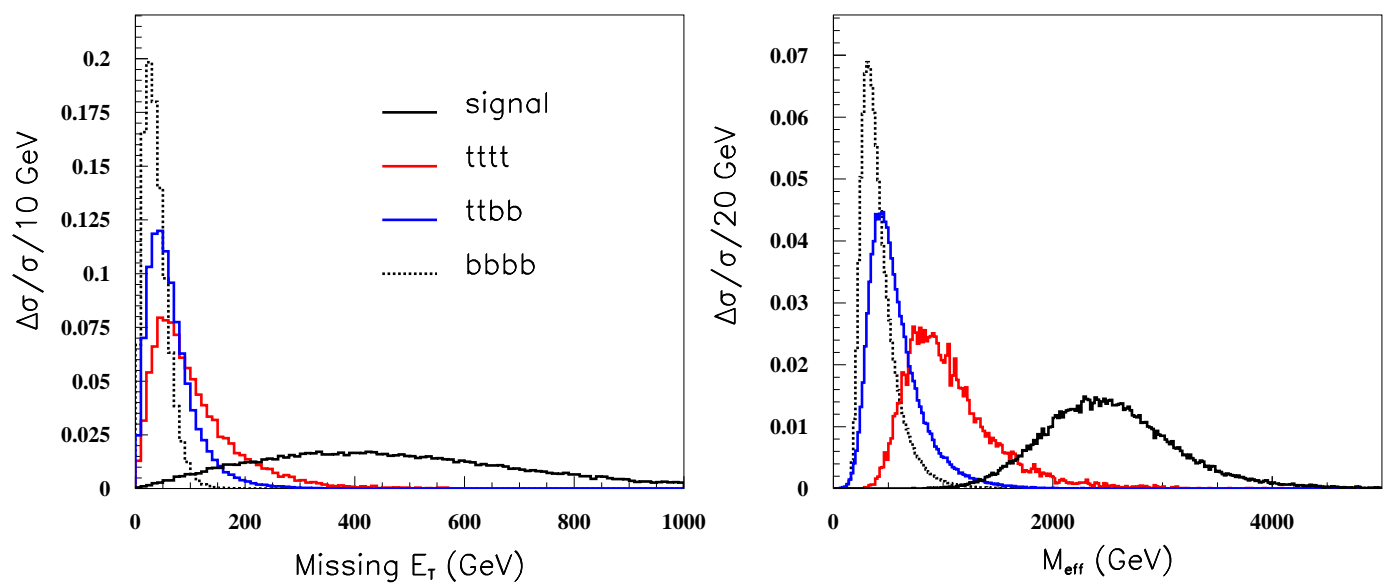

Figure 1: Distributions for missing transverse energy $\left(\mathrm{E}_{\mathrm{T}}\right)$ (left) and effective mass $\left(\mathrm{M}_{\mathrm{eff}}\right)$ (right) for four heavy flavor final states and signal with $m_{\tilde{\mathrm{g}}}=1751.3 \mathrm{GeV}$ ( FP1, see text): in both the figures the distributions are indicative of shapes only and normalized to unit cross section.

The distributions of $\mathbb{E}_{\mathrm{T}}$ and $\mathrm{M}_{\mathrm{eff}}$ for different final states involving four third generation quarks are shown in Fig. 1. Selection efficiencies for these background processes due to individual cuts are shown in Table 3; the individual cut which rejects most events for each final state is shown in bold. The residual cross-sections for these backgrounds after all our selection cuts are shown in Table 4. As is expected from Fig. 1 very little background survives.

For comparison we have computed the backgrounds corresponding to the cuts implemented by the ATLAS collaboration [18] (column 5 of Table 4) and Baer et al.[19] (column 6 of Table 4) . It may be noted that in all cases these backgrounds are rather small compared to other backgrounds from $t \bar{t}$ production or QCD processes (see below). 


\begin{tabular}{lllllll}
\hline \hline Process & $\mathrm{N}_{\text {jet }} \geq 6$ & $N_{o b j} \geq 9$ & $\mathrm{~N}_{\mathrm{btag}} \geq 2$ & $\begin{array}{l}\mathrm{P}_{\mathrm{T}}^{\mathrm{b} 1} \geq 300 \\
\mathrm{GeV}\end{array}$ & $\begin{array}{l}\mathrm{E}_{\mathrm{T}} \geq 300 \\
\mathrm{GeV}\end{array}$ & $\begin{array}{l}\mathrm{M}_{\text {eff }} \\
2000 \mathrm{GeV}\end{array}$ \\
\hline $\mathrm{t} \overline{\mathrm{t} t \overline{\mathrm{t}}}$ & 0.9304 & 0.5457 & 0.5946 & 0.1014 & $\mathbf{0 . 0 3 1 6}$ & $\mathbf{0 . 0 3 4 6}$ \\
$\mathrm{t} \overline{\mathrm{tb}} \overline{\mathrm{b}}$ & 0.5792 & 0.0831 & 0.4509 & 0.0265 & 0.0033 & $\mathbf{0 . 0 0 1 8}$ \\
$\mathrm{b} \overline{\mathrm{b}} \mathrm{b} \overline{\mathrm{b}}$ & 0.2186 & 0.0064 & 0.5854 & 0.0120 & $\mathbf{0 . 0 0 0 0 4}$ & 0.0002 \\
& & & & & & \\
\hline \hline
\end{tabular}

Table 3: Selection efficiencies for different final states involving four third generation quarks due to individual cuts have been shown. The individual cut which rejects most events for each background is shown in bold.

\begin{tabular}{lcc|ccc}
\hline \hline Process & $\begin{array}{c}\sigma_{\text {gen }} \\
(\mathrm{fb})\end{array}$ & $\begin{array}{c}\text { Events } \\
\text { generated }\end{array}$ & $\begin{array}{c}\text { This work } \\
\epsilon_{\text {sel }} \cdot \sigma_{\text {gen }}\end{array}$ & $\begin{array}{c}\text { ATLAS } \\
\epsilon_{\text {sel }} \cdot \sigma_{\text {gen }}\end{array}$ & $\begin{array}{c}\text { Baer } \text { et al. } \\
\epsilon_{\text {sel }} \cdot \sigma_{\text {gen }}\end{array}$ \\
\hline $\mathrm{t} \overline{\mathrm{t} \overline{\mathrm{t}}}$ & 5.3 & $1.25 \times 10^{4}$ & 0.012 & 0.093 & 0.004 \\
$\mathrm{t} \overline{\mathrm{tb}} \mathrm{b}$ & 831 & $1.40 \times 10^{5}$ & 0.042 & 0.665 & 0.059 \\
$\mathrm{~b} \bar{b} \bar{b}$ & 7260 & $3.02 \times 10^{5}$ & 0.000 & 0.240 & 0.000 \\
& & & & & \\
\hline \hline
\end{tabular}

Table 4: The cross section at the generation level $\left(\sigma_{\text {gen }}\right)$, number of events generated, and the residual cross-section $\left(\epsilon_{\mathrm{sel}} \cdot \sigma_{\mathrm{gen}}\right)$ after all cuts. The cross sections were computed with cuts on final states partons different from the ones used in Table 2 and the default QCD scale $\left(\mathrm{Q}^{2}=\Sigma_{\mathrm{f}}\left(\mathrm{m}_{\mathrm{f}}^{2}+\mathrm{P}_{\mathrm{T}_{\mathrm{f}}}^{2}\right)\right.$, where ' $\mathrm{f}^{\prime}$ stands for the final states partons) of ALPGEN. 
This conclusion holds even if a factor of two due to the QCD scale uncertainty is taken into account. It may be recalled that the cuts in [18] are designed for relatively light gluinos in the focus point region. Consequently they are less severe than the ones introduced in this paper or in [19]. As a result some of the residual backgrounds of this type are numerically significant for integrated luminosities $\geq 100 \mathrm{fb}^{-1}$ and require some attention.

Next we turn our attention to the conventional backgrounds. First we shall summarize the earlier works which employed b-tagging. Mercadante et al.[15] found that the dominant background comes from QCD processes involving light flavors and gluons only. The analysis of reference [18] ignored this background arguing qualitatively that the requirement of 2-tagged b-jets should be adequate to reduce it to a negligible level. However, they studied the $\mathrm{b} \bar{b} j$ background, which is included in the QCD background in this work as well as in [19] and found it to be $41 \%$ of the dominant t $\bar{t}$ background. Very recently Baer et al.[19] analyzed the same background with different kinematical cuts. They found that this background is rather small.

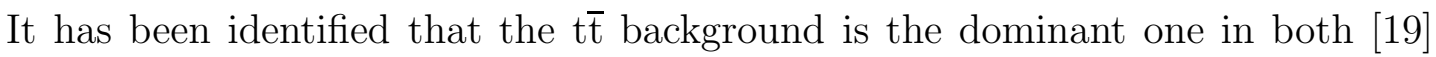
and [18] and we agree with them qualitatively. In the former work this background was simulated by ISAJET [28]. However, in addition to other selection criteria they required $>7$ jets $+>2$ tagged b-jets in the final state. Since the parton level process has at most six jets (including two b-jets) the size of this background crucially depends on the model of parton showering in the generator. Our cut on $\mathrm{N}_{\text {obj }}$ is similar in nature. We shall briefly comment on the reliability of analyses requiring high jet multiplicity in the following.

We now present our estimates of these backgrounds. The cross-sections for QCD processes (which includes $\mathrm{b} \overline{\mathrm{b}}$ and $c \bar{c}$ production) and $\mathrm{t} \overline{\mathrm{t}}$ production are quite large (see Table 6). Since the selection demands a large number of jets, $\mathrm{E}_{\mathrm{T}}$ and $\mathrm{M}_{\text {eff }}$ it is more likely that events with higher $\sqrt{ } \hat{s}$ will survive. Even then both the above backgrounds survive the cuts (mainly the one on the multiplicity of objects) because of initial state and final state radiation (ISR and FSR) and multiple interaction. On the other hand events with lower $\sqrt{ } \hat{s}$ have a very large share of the cross-section and ISR and FSR help them to pass the selection. To estimate the contributions from the above two regions with sufficient statistics and to avoid generating large number of events which will not pass the selection, we have generated events with certain cuts at the parton level:

- $\mathrm{t} \overline{\mathrm{t}}$ events have been generated in two bins, $\hat{\mathrm{P}_{\mathrm{T}}^{\mathrm{t}}} \leq 600 \mathrm{GeV}$ (tE-low) and $\hat{\mathrm{P}_{\mathrm{T}}^{\mathrm{t}}}>600$ $\mathrm{GeV}$ (t⿱t-)-high).

- $\mathrm{QCD}$ events have also been generated in two bins, $100 \mathrm{GeV} \leq \hat{\mathrm{P}_{\mathrm{T}}} \leq 600 \mathrm{GeV}$ (QCD-low) and $\hat{\mathrm{P}_{\mathrm{T}}}>600 \mathrm{GeV}$ ( QCD-high) for the final state partons.

The efficiency of the individual cuts and the size of the different backgrounds are listed separately in Tables 5 and 6 . The cross sections are calculated with the conservative choice of the QCD scale. It is to be noted that while the cut $\mathrm{N}_{\mathrm{obj}} \geq 9$ is most effective for rejecting tt-high events with high $\sqrt{ } \hat{\mathrm{s}}$, it is either the cut on $\mathbb{E}_{\mathrm{T}}$ or $\mathrm{M}_{\text {eff }}$ for events with low 
$\sqrt{ } \hat{\mathrm{s}}$. The $\mathbb{T}_{\mathrm{T}}$ distributions before and after applying all other kinematical cuts are shown in Fig. 2. The distributions of $\mathrm{N}_{\text {jet }}$ and $\mathrm{N}_{\mathrm{obj}}$ are shown in Fig. 3. It is obvious that even after the cut $\mathrm{N}_{\text {jet }}>7$ quite a bit of background survives where as the cut $\mathrm{N}_{\text {obj }}>9$ suppresses the background more effectively.
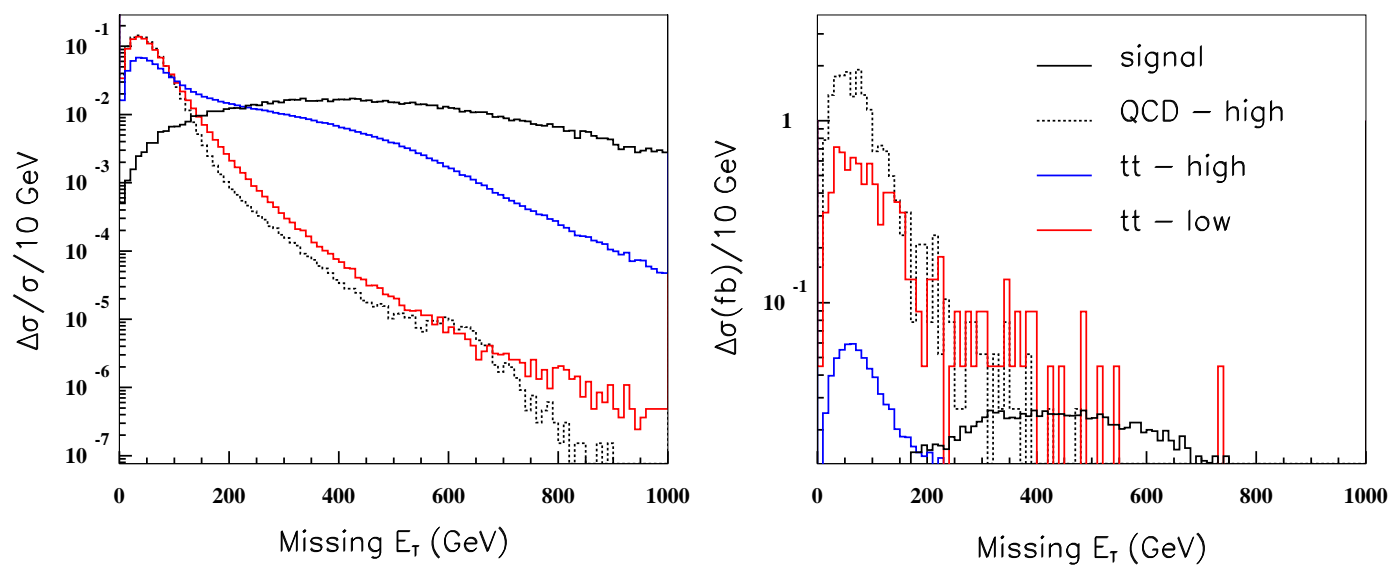

Figure 2: Missing transverse energy ( $\mathbb{E}_{T}$ ) distributions for the major backgrounds and the signal $\left(\mathrm{m}_{\tilde{\mathrm{g}}}=1751.3 \mathrm{GeV}\right)$. In the left plot the distributions are indicative of the shapes only. In the right plot they have been normalized to the residual cross-sections after all cuts except the one on $\mathrm{E}_{\mathrm{T}}$.

We do not impose any extra strong hardness cut on a jet which is counted as an acceptable object. It is quite plausible that in a real life situation such jets indeed arise from showering. However as noted before the number of such jets in an event crucially depends on the parton showering model in the generator. Therefore the reliability of the background estimate both in this work as well as in [19] can not be guaranteed until the showering model is validated using LHC data. In the next paragraph we briefly summarize a study [29] which indicates that the t⿹ background estimated as above may not be wide off the mark.

It is certainly desirable to supplement the lowest order estimates of the background by higher order matrix element calculations. However, care must be taken to avoid double counting of jets which may arise in a lower order calculation due to hard emissions during shower evolution as well as from a higher order matrix element. A proper matching of matrix elements and shower evolution is, therefore, called for. In reference [29] the lowest order cross section of $t \bar{t}$ production was computed. Next the combined cross section of the processes t $\bar{t}$ (exclusive), $t \bar{t} j$ (exclusive), $t \bar{t} j j$ (exclusive)and $t \bar{t} j j j$ (inclusive), where $\mathrm{j}$ stands for a light quark or gluon, were obtained after matrix element matching( see Table 1 and Table 2 of [29]). The two results agree nicely both for Tevatron and LHC energies. A similar agreement is found for various distributions of the produced $t \bar{t}$ system computed by the two different ways. It is these distributions which after all determine the efficiencies of various cuts. Thus one may conclude that at least the lowest order $t \bar{t}$ 

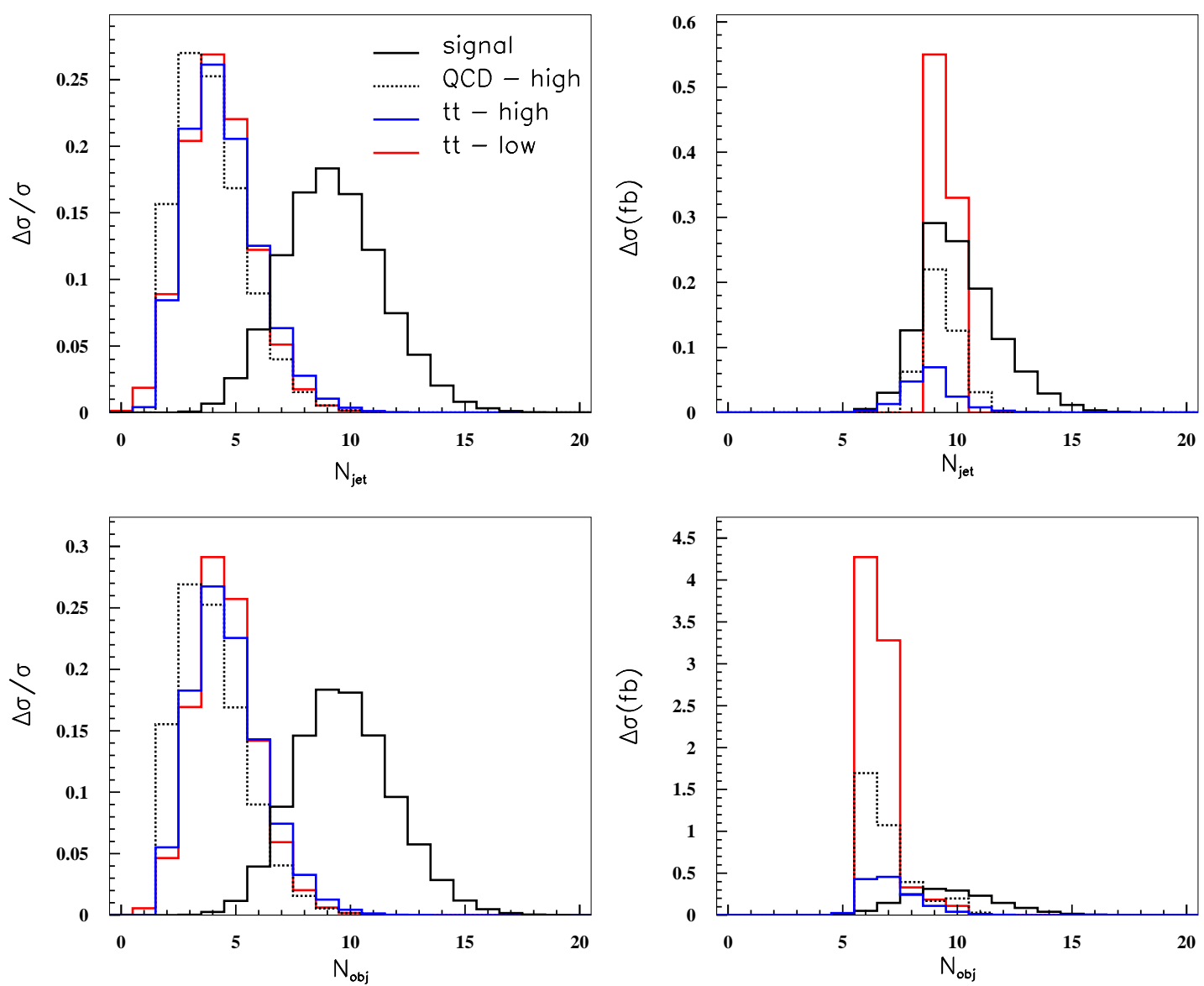

Figure 3: The distributions of the number of jets $\left(\mathrm{N}_{\text {jet }}\right)$ (top) and number of objects $\left(\mathrm{N}_{\text {obj }}\right)$ (bottom) for the major backgrounds and the signal $\left(\mathrm{m}_{\widetilde{\mathrm{g}}}=1751.3 \mathrm{GeV}\right)$. The figures on the left show the distributions before any cut (normalized to unit cross section); the figures on the right show distributions normalized to residual cross-sections after all cuts except those on $\mathrm{N}_{\text {jet }}$ (top) and $\mathrm{N}_{\mathrm{obj}}$ (bottom). 


\begin{tabular}{lllllll}
\hline \hline Process & $N_{\text {jet }} \geq 6$ & $\mathrm{~N}_{\text {obj }} \geq 9$ & $\mathrm{~N}_{\text {btag }} \geq 2$ & $\begin{array}{l}\mathrm{P}_{\mathrm{T}}^{\mathrm{b1}} \geq 300 \\
\mathrm{GeV}\end{array}$ & $\begin{array}{l}\mathrm{E}_{\mathrm{T}} \geq 300 \\
\mathrm{GeV}\end{array}$ & $\begin{array}{l}\mathrm{M}_{\text {eff }} \geq \\
2000 \mathrm{GeV}\end{array}$ \\
\hline Signal & 0.9674 & 0.7116 & 0.7899 & 0.6275 & 0.7416 & 0.8173 \\
QCD - high & 0.1490 & 0.0074 & 0.0177 & 0.0599 & $\mathbf{0 . 0 0 1 8}$ & 0.1033 \\
QCD - low & 0.0151 & 0.0001 & 0.0052 & 0.0003 & $\mathbf{0 . 0 0 0 0}$ & $1.9 \times 10^{-6}$ \\
$\mathrm{t} \bar{t}$ - high & 0.2318 & $\mathbf{0 . 0 1 8 8}$ & 0.4567 & 0.5853 & 0.1799 & 0.0963 \\
$\mathrm{t} \bar{t}$ - low & 0.1984 & 0.0083 & 0.1490 & 0.0074 & 0.0023 & $\mathbf{0 . 0 0 1 4}$ \\
\hline \hline
\end{tabular}

Table 5: Selection efficiencies for major backgrounds due to individual cuts. The individual cut which rejects most events for each case is shown in bold.

\begin{tabular}{|c|c|c|c|}
\hline \multirow[t]{2}{*}{ Process } & \multirow{2}{*}{$\begin{array}{l}\sigma_{\text {gen }} \\
(\mathrm{fb})\end{array}$} & \multirow{2}{*}{$\begin{array}{c}\text { Events } \\
\text { generated }\end{array}$} & Residual cross-section $\left(\epsilon_{\text {sel }} \cdot \sigma_{\text {gen }}\right)$ \\
\hline & & & $\mathrm{N}_{\text {btag }} \geq 2$ \\
\hline
\end{tabular}

Signal

\begin{tabular}{lllll}
$\mathrm{m}_{\widetilde{\mathrm{g}}}=1751.3$ & 1.20 & $10^{4}$ & 0.3598 & 0.2445 \\
$\mathrm{~m}_{\widetilde{\mathrm{g}}}=1824.7$ & 0.81 & $10^{4}$ & 0.2267 & 0.1527 \\
$\mathrm{~m}_{\widetilde{\mathrm{g}}}=1950.0$ & 0.40 & $10^{4}$ & 0.1445 & 0.0987 \\
$\mathrm{~m}_{\widetilde{\mathrm{g}}}=2047.4$ & 0.24 & $10^{4}$ & 0.0921 & 0.0634 \\
$\mathrm{~m}_{\widetilde{\mathrm{g}}}=2119.8$ & 0.16 & $10^{4}$ & 0.0652 & 0.0431 \\
\hline
\end{tabular}

Background

\begin{tabular}{|c|c|c|c|c|}
\hline QCD - high & $1.81 \times 10^{5}$ & $1.08 \times 10^{7}$ & 0.4362 & 0.0504 \\
\hline t $\bar{t}-$ high & $4.14 \times 10^{2}$ & $2.325 \times 10^{6}$ & 0.1675 & 0.0205 \\
\hline $\mathrm{t} \overline{\mathrm{t}}-\mathrm{low}$ & $3.70 \times 10^{5}$ & $1.14 \times 10^{7}$ & 0.8806 & 0.1793 \\
\hline
\end{tabular}

Table 6: The cross-section at the generation level $\left(\sigma_{\mathrm{gen}}\right)$, number of events generated, and residual cross-section $\left(\epsilon_{\text {sel }} \cdot \sigma_{\text {gen }}\right)$ after all cuts are shown. $\sigma_{\text {gen }}$ is calculated for $\mathrm{Q}^{2}=\hat{\mathrm{s}}$ 

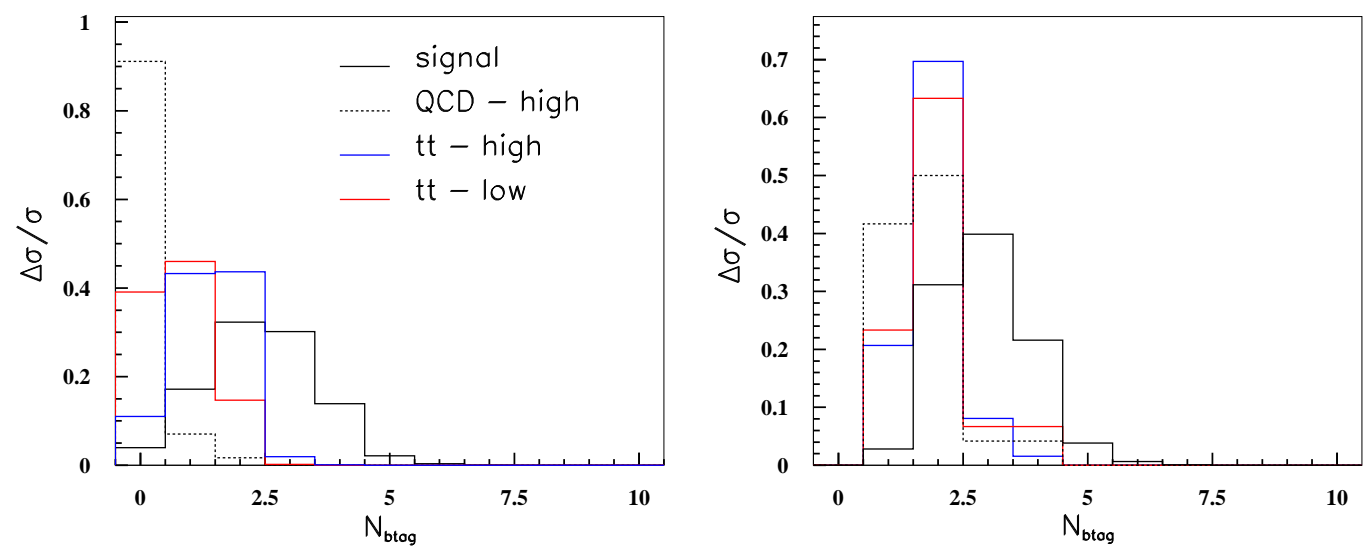

Figure 4: Distributions for number of b-tagged jets ( $\mathrm{N}_{\mathrm{btag}}$ ) are shown: (left) before any selection criteria has been applied and (right) after other selection criteria have been applied. In both the distributions are indicative of shapes only.

background including parton showering which is the dominant background for the signal under study is fairly reliable.

The case of the pure light flavor QCD background is rather similar. The required final state with nine objects from this $2 \rightarrow 2$ process can arise solely due to parton showering and, therefore, model dependent to some extent. Simulating the lowest order QCD processes in Pythia we find that the size of this background is smaller than the t $\bar{t}$ background but is non-negligible. However the entire contribution comes from QCD-high events $\hat{\mathrm{P}_{\mathrm{T}}}>600 \mathrm{GeV}$. After generating 15 million QCD-low $\left(100 \mathrm{GeV} \leq \hat{\mathrm{P}_{\mathrm{T}}} \leq 600 \mathrm{GeV}\right)$ events we found that no event survive the cuts.

We now compare our results with other calculations which required two or more tagged b-jets in the final state. A comparison with [18] may not be meaningful as their cuts are designed for the search of a relatively light gluino $\left(\mathrm{m}_{\widetilde{\mathrm{g}}}=857.0\right)$. In fact with their cuts we find that the survival probability of the tE background is twice as large as our estimate and will overwhelm the gluino signal for $\mathrm{m}_{\widetilde{\mathrm{g}}} \approx 2 \mathrm{TeV}$, which is roughly the $\mathrm{LHC}$ reach in the FP region, having a cross section not exceeding a few fb's.

The t€ background after all cuts estimated by Baer et al.[19] is $1.5 \mathrm{fb}$ with 'a tiny' contribution from QCD. On the other hand we get a smaller t $\bar{t}$ background while the QCD background is non-negligible. Thus one may conclude that our cuts are more efficient in reducing the former background while the cuts of [18] may be better for eliminating the QCD backgrounds. A more detailed comparison is not possible since we donot know the QCD scales for different cross sections in [19].

It is probably a numerical conspiracy that the total background ( $\mathrm{t} \overline{\mathrm{t}}+\mathrm{QCD}$ ) computed by the two groups agree rather nicely. However, the fact that two groups using 
different event generators and different sets of kinematical cuts get comparable results at least indicates that the major backgrounds obscuring the search for focus point SUSY at LHC are fairly well understood.

We next present the signal size for different gluino masses. The efficiencies of the cuts used in this paper for the scenario FP1 are in Table 5 while the signal size for the conservative scale choice in different scenarios after all cuts are in Table 6 . The signal cross sections should be multiplied by a factor of 2 if the scale $\mathrm{m}_{\tilde{\mathrm{g}}} / 2$ is considered (see Table 1). The $\mathrm{E}_{\mathrm{T}}, \mathrm{N}_{\text {jet }}$ and $\mathrm{N}_{\mathrm{obj}}$ distributions are in Figs. 2 and 3. Comparing these with the background cross sections and taking the QCD scale uncertainty into account it is clear that the gluino mass reach at the LHC cannot be pin pointed as yet. Nevertheless the following comments are in order.

Using the optimistic scale choice $\left(=\mathrm{m}_{\tilde{\mathrm{g}}} / 2\right)$ one finds that for FP1(FP3) (corresponding to $\left.\mathrm{m}_{\tilde{\mathrm{g}}}=1751.3(1950)\right) \mathrm{S} / \sqrt{B}=10.0$ (4.0) with an integrated luminosity 300 $\mathrm{fb}^{-1}$, where $\mathrm{S}$ and $\mathrm{B}$ are the number of signal and background events respectively. Of course the $\mathrm{S} / \sqrt{B}$ ratio may be modestly increased by optimizing the cuts [15]. On the other hand the LO signal cross section at the scale $\mathrm{m}_{\widetilde{\mathrm{g}}} / 2$ mimics the NLO cross section [24]. In view of this the next to leading order $t \bar{t}$ cross section (about a factor of 2 larger than the cross section in Table 6 ) may give a more reliable background estimate. This will further suppress the $\mathrm{S} / \sqrt{B}$ ratio by a factor of $\sqrt{2}$. For the conservative scale choice on the other hand the above ratios will be suppressed by a factor of two. On the whole it seems unlikely that using the selection criteria based on 2 or more tagged b-jets as is usually required, the gluino mass reach at LHC can be pushed far beyond $1.8 \mathrm{TeV}$ with certainty.

We now impose a more stringent b-identification criteria as suggested in [12] and require $\geq 3$ b-jet tags keeping all other selection criteria listed above unchanged. The residual signal and the backgrounds are shown in the last column of Table 6 . Even the conservative scale choice now yields $\mathrm{S} / \sqrt{B}$ ratio 8.5 (3.4) for $\mathrm{m}_{\widetilde{\mathrm{g}}}=1751.3$ (1950). For the optimistic scale choice the above ratios are enhanced approximately by a factor of $\sqrt{2.0}$ after doubling the background which should reflect the theoretical uncertainties due to possible NLO corrections etc. In view of the possibility of further improving this ratio by optimizing the selection criteria, one can hope that irrespective of the uncertainty due to the choice of scale the reach in $\mathrm{m}_{\widetilde{\mathrm{g}}}$ at $\mathrm{LHC}$ is close to $2.0 \mathrm{TeV}$.

\section{Conclusion}

If supersymmetry in the focus point region is realized in nature it may lead to spectacular signals at the LHC via gluino pair production $[12,13,15,16,17,18,19]$. We propose a new set of criteria for selecting(rejecting) the signal( background) (see section 2).

We have extended the estimation of the backgrounds which may hinder the search

for supersymmetry in the focus point region. In particular we have simulated and ana- 
lyzed the potentially dangerous backgrounds from different processes involving four heavy flavor quark production which have so far been neglected. Some of these processes have cross sections several orders of magnitude larger than that of the signal. Moreover these backgrounds are rich in b-jets and cannot be reduced by b-tagging which is recommended as one of the most effective tools for removing other SM backgrounds [12, 15, 19].

Using rather stringent cuts (see section 2) appropriate for detecting relatively heavy gluinos with masses near the kinematic reach of the LHC, we have shown that these background are highly suppressed(see Table 4). On the other hand for less severe cuts often employed for relatively light gluinos in the focus point region (see for example [18]), these backgrounds may be small but numerically significant and call for some attention.

We have also simulated the conventional SM backgrounds estimated by other groups. We qualitatively agree with the observation $[18,19]$ that t $\bar{t}$ events are the most severe background to the gluino signal. In view of various theoretical uncertainties in the production cross sections the LHC reach in $\mathrm{m}_{\widetilde{\mathrm{g}}}$ cannot be pinpointed as yet. This is especially so if the selection criteria is restricted to $\geq 2$ tagged b-jets which is the usual strategy. Requiring 3 or more tagged b-jets in the signal the gluino mass reach at LHC is close to $2.0 \mathrm{TeV}$ even with a modest production cross section corresponding to a conservative choice of the QCD scale.

We look forward to improved b-tagging efficiencies along with improvement in the theoretical understanding (e.g., the inclusion of matrix element matching) of the light flavor QCD and other backgrounds for a better handle on gluino searches in the FP scenario.

Acknowledgement: SPD acknowledges postdoctoral fellowships from Bundesministerium für Bildung und Forschung (BMBF) Projekt under Contract No. 05HT6PDA and Harish Chandra Research Institute where part of this work was done. $\mathrm{AD}$ and MM acknowledge support by the DST, India (project SR/S2/HEP-18/2003). MM and SM acknowledge support by the DST, India (project SP/S2/K-25/96-VI). The authors thank M. Drees, H. Driener, M.L. Mangano and S. Mrenna for many useful suggestions and discussions.

\section{References}

[1] For reviews on Supersymmetry, see, e.g., H. P. Nilles, Phys. Rep. 1, 110 ( 1984); H. E. Haber and G. Kane, Phys. Rep. 117, 75 ( 1985) ; J. Wess and J. Bagger, Supersymmetry and Supergravity, 2nd ed., (Princeton, 1991); M. Drees, P. Roy and R. M. Godbole, Theory and Phenomenology of Sparticles, (World Scientific, Singapore, 2005).

[2] K. L. Chan, U. Chattopadhyay and P. Nath, Phys. Rev. D 58, 096004 (1998); J. Feng, K. Matchev and T. Moroi, Phys. Rev. Lett. 84, 2322 (2000), Phys. Rev. D 61, 075005 (2000). 
[3] For a review see, e.g., J. Feng and F. Wilczek, Phys. Lett. B 631, 170 (2005).

[4] A. H. Chamseddine, R. Arnowitt and P. Nath, Phys. Rev. Lett. 49, 970 ( 1982); R. Barbieri, S. Ferrara and C. A. Savoy, Phys. Lett. B 119, 343 (1982); L. J. Hall, J. Lykken and S. Weinberg, Phys. Rev. D 27, 2359 (1983); P. Nath, R. Arnowitt and A. H. Chamseddine, Nucl. Phys. B 227, 121 (1983); N. Ohta, Prog. Theor. Phys. 70, 542 (1983); For reviews see, e.g., [1] and P. Nath, R. Arnowitt and A.H. Chamseddine, Applied $N=1$ Supergravity (World Scientific, Singapore, 1984).

[5] J.L. Feng and K. T. Matchev, Phys. Rev. D 63, 0950003 (2000).

[6] R. Barbieri and G. F. Giudice, Nucl. Phys. B 306, 63 (1988); B. de Carlos and J. A. Casas, Phys. Lett. B 309, 320 (1993) ; G. W. Anderson and D. J. Castano, Phys. Lett. B 347, 300 (1995) ; Phys. Rev. D 52, 1693 (1995); Phys. Rev. D 53, 2403 (1996); P. Ciafaloni and A. Strumia, Nucl. Phys. B 494, 41 (1997); G. Bhattacharyya and A. Romanino, Phys. Rev. D 55, 7015 (1997) ; P. H. Chankowski, J. Ellis and S. Pokorski, Phys. Lett. B 423, 327 (1998).

[7] M. Drees, M.M. Nojiri, D.P. Roy and Y. Yamada, Phys. Rev. D 56, 276 (1997); Erratum-ibid. D64, 039901 (2001).

[8] J. Edsjö and P. Gondolo, Phys. Rev. D 56, 1879 (1997).

[9] J.L. Feng, K. T. Matchev and F. Wilczek, Phys. Lett. B 482, 388 (2000); Phys. Rev. D 63, 045024 (2001).

[10] For a review, see, e.g., M. Drees, AIP Conf. Proc. 805, 48 (2006), arXiv:hep-ph/0509105.

[11] See D. N. Spergel et al., Astrophys. J. Suppl. 170, 377 (2007), arXiv:astro-ph/0603449 and references therein.

[12] U. Chattopadhyay, Amitava Datta, A. Datta, A. Datta and D. P. Roy, Phys. Lett. B 493, 127 (2000).

[13] H. Baer, A. Belyaev, T. Krupovnickas and X. Tata, J. High Energy Phys. 0306, 054 (2003).

[14] H. Baer, T. Krupovnickas, S. Profumo and P. Ullio, J. High Energy Phys. 0510, $020(2005)$.

[15] P. G. Mercadante, J. K. Mizukoshi and X. Tata, Phys. Rev. D 72, 035009 (2005).

[16] D. Denegri et al.Nuovo cim 120B, 687 (2005).

[17] A. Belyaev, Int. J. Mod. Phys. A 21, 205 (2006).

[18] U. de Sanctis, T. Lari, S. Montesano, C. Troncon, ATLAS-SCIENTIFIC-NOTE-SNATLAS-2007-062, Apr 2007; arXiv:0704.2515 [hep-ex]. 
[19] H. Baer et al., Phys. Rev. D 75, 095010 (2007).

[20] See, e.g., A.Pukhov, "CalcHEPv2.4.5 - a package for evaluation of Feynman diagrams and integration over multi-particle phase-space", see the URL: http://www.ifh.de/ pukhov/calchep.html.

[21] M. L. Mangano, M. Moretti, F. Piccinini, R. Pittau and A. Polosa, J. High Energy Phys. 0307, 001 (2003).

[22] See e.g., F. Maltoni and T. Stelzer, J. High Energy Phys. 0302, 027 (2003).

[23] T. Sjostrand, S. Mrenna and P. Skands, J. High Energy Phys. 0605, 026 (2006).

[24] W. Beenakker et al., Nucl. Phys. B 492, 51 (1997).

[25] The recent world average value for the top quark mass is $m_{t}=170.9 \mathrm{GeV}[26]$. We have, however, chosen the reference value $m_{t}=175 \mathrm{GeV}$. This facilitates the comparison with other published studies.

[26] P. A. M. Fernandez for the CDF and D0 collaborations, arXiv:0705.3910 [hep-ex]; M. H. L. S. Wang for the CDF, D0 collaborations, arXiv:0705.3873 [hep-ex].

[27] CTEQ collaboration, H. L. Lai et al., Phys. Rev. D 55, 1280 (1997).

[28] F. E. Paige, S. D. Protopopescu, H. Baer and X. Tata, hep-ph/0312045.

[29] M. L. Mangano, M. Moretti, F. Piccinini and M. Treccani, J. High Energy Phys. 0701, 013 (2007). 